\title{
Promotion of the Stability of Lycopene by Liposomal formulations for Anti-aging Therapy
}

\section{Hoang Van Luong ${ }^{\star}$}

Vice-director, Vietnam Military Medical University, Vietnam

*Corresponding author: Hoang Van Luong, Vice-director, Vietnam Military Medical University, Vietnam, Tel: (84) 69566103; E-mail: luonghv@vmmu.edu.vn Rec date: September 19, 2014, Acc date: September 22, 2014, Pub date: September 28, 2014

Copyright: (c) 2014 Luong VH. This is an open-access article distributed under the terms of the Creative Commons Attribution License, which permits unrestricted use, distribution, and reproduction in any medium, provided the original author and source are credited.

\section{Editorial}

Reactive oxygen species (ROS), including hydrogen peroxide, hydroxyl radical, and superoxide anion can be created as usual products of aerobic metabolism and can be formed at elevated rates under pathophysiological conditions. Overproduction of ROS leads to significant damages to cell structure and functions and could be the cause of aging. Oxidative stress (OS) is defined as an imbalance between the production of ROS and antioxidant defenses that can result in cellular and tissue damage [1,2]. Subsequently, the potential strategy in preventing oxidant-induced cellular and tissue damage involves the use of appropriate antioxidants. Antioxidants are substances capable of preventing, inhibiting, or slowing the oxidation of other molecules [3,4]. In vitro studies showed that when applied directly and at high concentrations of antioxidants to cellular systems, they are effective in conferring protection against the damaging actions of ROS. However, using antioxidants in therapies has limitation of their poor solubility, instability, inability to cross membrane barriers and rapid clearance from cells. Therefore, there is considerable interest towards the development of antioxidant-delivery systems that would result in the stability, solubility and selective delivery of the compounds to tissues in sufficient concentrations to ameliorate injured tissues caused by oxidant.

\section{Carotenoids as antioxidants}

Recent work from our lab has focused on carotenoids and the use of liposomes for the delivery of some carotenoids such as lycopene and astaxanthin as antioxidants in the prevention or treatment of several pathological conditions related to oxidative stress. Lycopene possesses an antioxidative capability much stronger than zeaxanthin, lutein and $\beta$-carotene. However, its use is often limited due to its instability, poor water solubility and very low bioavailability following topical and oral administration.

The structure of lycopene consists of 13 double-bonds. Two of them are non-conjugated, whereas eleven are conjugated double bonds, thus building a chromophore. This conjugated polyene structure accounts for the red color and the antioxidant properties of lycopene [5]. Lycopene is susceptible to chemical changes such as oxidation followed by degradation or isomerization when exposed to light, heat and oxygen. Our study revealed that crystal lycopene and lycopene in chloroform were shown to be degraded completely under laboratory condition $\left(25^{\circ} \mathrm{C}\right)$ after 3 weeks and 6 days, respectively. In process of degradation, several chemical reactions can induce isomerization from the trans-isomer to several mono- or poly-cis forms [5]. The forms of lycopene detected in human serum and tissues range from all-trans to 9-, 13- and 15-cis isomers, whereas the predominant lycopene in food is the all-trans form.

\section{Lycopene liposomes}

In order to improve the stability of antioxidants, liposomal formulations are being developed [6]. Liposome delivery systems are potential candidates for biocompatible, biodegradable, and nontoxic artificial phospholipid vesicles that offer the possibility of carrying hydrophilic, hydrophobic molecules. In our study, lycopene liposomes are prepared using lecithin (3\%), cholesterol $(0.3 \%)$, and water phase of $74.5 \%$. In the formulation, lycopene can be incorporated into the lipid bilayer of the liposome $[7,8]$. Incorporation of lycopene into liposomes not only reduced lipid damage but also slowed the oxidation of lycopene [9].

Our experiment showed that lycopene in liposomes was stable for at least 3 months at room conditions. Further studies on solubility revealed that lycopene can encapsulated up to concentration of 200 $\mu \mathrm{g} / \mathrm{ml}$ with menthyl anthranilate as the solvent. Moreover, liposomes containing ascorbic acid showed a significant stability improvement of lycopene. The ability for precise adjustments of lycopene liposome parameters such as size, charge, lipid composition, and the conjugation of ligands coupled with the more efficient and loading techniques offers the possibility to make liposomes an effective platform for the delivery of lycopene.

In summary, lycopene in liposomal formulations could be stable, and hold great promises in the treatment of many diseases in which oxidative stress plays a significant role. Liposomes are highly efficient in terms of facilitating antioxidant delivery and achieving prophylactic and therapeutic efficacies against oxidative stress-induced damage and aging.

\section{References}

1. Ward PA (2010) Oxidative stress: acute and progressive lung injury. Annals of the New York Academy of Sciences 1203: 53-59.

2. Ziech D, Franco R, Georgakilas G et al. (2010) The role of reactive oxygen species and oxidative stress in environmental carcinogenesis and biomarker development. Chem biol interact 188: 334-339.

3. Suntres ZE (2002) Role of antioxidants in paraquat toxicity. Toxicology 180: 65-77.

4. Sies H (1997) Oxidative stress: oxidants and antioxidants. Exp Physiol 82, 2: 291-295.

5. Shi J, Le Maguer M (2000) Lycopene in tomatoes: Chemical and physical properties affected by food processing. Crit. Rev. Biotechnol. 20: 293-334.

6. Stone WL, Smith M (2004) Therapeutic uses of antioxidant liposomes. Mol biotechnol 3: 217-230.

7. Schuber F, Kichler A, Boeckler C, Frisch B (1998) Liposomes: from membrane models to gene therapy. Pure and Applied Chemistry 1: 89-96.

8. Samad A, Sultana Y, and Aqil M (2007) Liposomal drug delivery systems: an update review. Current Drug Delivery 4: 297-305. 
Citation: Luong HV (2014) Promotion of the Stability of Lycopene by Liposomal formulations for Anti-aging Therapy. Aging Sci 2: e113. doi: 10.4172/2329-8847.1000e113

Page 2 of 2

9. Lautenschlager H, Barel AO, Paye M, Maibach HI (2006) Liposomes, Handbook of Cosmetic Science and Technology. CRC Press Taylor \& Francis Group, Boca Raton: 155-163. 